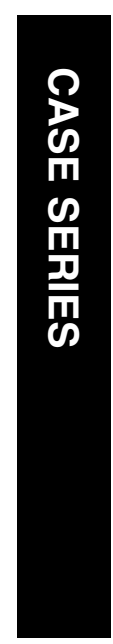

\section{Two cases of acute macular neuroretinopathy}

\begin{abstract}
Purpose To report chorioretinal vasoconstriction as a potential pathogenic mechanism in acute macular neuroretinopathy (AMNR). To describe a time lag between the onset of functional deficits and that of fundoscopically visible lesions and illustrate the superior value of infrared (IR) compared to red-free or white light imaging in AMNR. Methods Two young female patients (30 and 19 years old) with AMNR are described. Both underwent detailed clinical examination with additional imaging using IR, blue, and red-free light. Functional evaluation with pattern and multifocal electroretinography, Goldmann manual, and automated Humphrey visual fields (VFs) was also performed.
\end{abstract}

Results The first patient was diagnosed with AMNR after a caesarian section during and after which she received treatment with vasoconstrictive drugs. She was followed up for 28 months, after which time she still suffered from bilateral U-shaped paracentral scotomata associated with macular lesions. The second patient complained of central scotomata prior to the onset of any visible fundoscopic lesions, following a bout of flu. VFs confirmed a central scotoma and pattern electroretinography was consistent with loss of macular function. Bilateral petaloid lesions became visible after 3 days when function began to improve. In both patients IR imaging was superior to standard red-free and white light in identifying macular lesions.

Conclusions Vasoconstriction in the chorioretina may be pathogenic in AMNR. Functional complaints precede fundus lesions in AMNR. And, IR light is superior to red-free or white light imaging in detecting typical fundus lesions in AMNR both early and late in the course of the disease.

Eye (2007) 21, 1226-1229; doi:10.1038/sj.eye.6702543; published online 15 September 2006
HD Corver, J Ruys, A-M Kestelyn-Stevens, J-J De Laey and BP Leroy

Keywords: acute macular neuroretinopathy; chorioretinal ischaemia; viral infection; infrared imaging; pattern electroretinography

\section{Introduction}

Acute macular neuroretinopathy (AMNR) is a rare condition first described in 1975 by Bos and Deutman. ${ }^{1}$ It is characterised by subacute visual impairment that can either be transient or permanent. Patients generally present with disturbance or loss of central vision and/or acute central or paracentral scotomata in either one or both eyes. These visual field (VF) defects correspond to petaloid macular lesions, best seen with red-free or infrared (IR) light. ${ }^{2,3}$ Several risk factors have been reported, ranging from viral illness and the use of epinephrine to acute episodes of hypotension. ${ }^{4}$ The lesions may be located in the outer neurosensory retina as suggested by optical coherence tomography (OCT) and multifocal electroretinography (mfERG). ${ }^{5-8}$

Here, we describe two young females with AMNR.

\section{Case reports}

Case 1 is that of a 30-year-old woman who had undergone a caesarian (C)-section under epidural anaesthesia with ropivacaine, and general relaxation with sufentanil. The patient had undergone two uncomplicated C-sections previously. She had a history of hypotension with blood pressures of around 100/60 $\mathrm{mmHg}$. A brief spell of unconsciousness, without a further apparent drop in blood pressure, occurred immediately after the epidural anaesthesia. After childbirth, methylergometrin was administered intravenously twice and a third time orally. Additionally, oral etilephrine was given $8 \mathrm{~h}$ later, and during subsequent days, because blood pressure failed to rise.

Immediately following $\mathrm{C}$-section, the patient complained of seeing black spots in the 
a

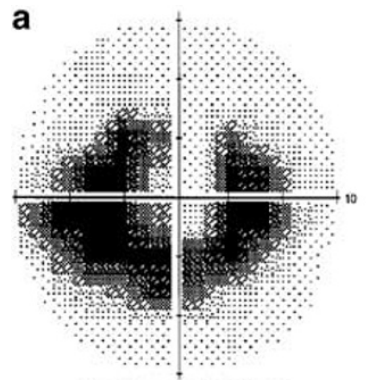

At presentation

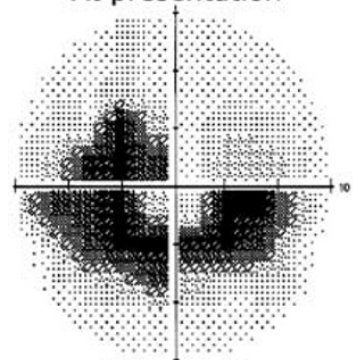

At 5 months

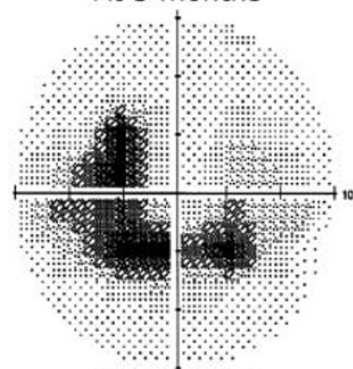

At 9 months

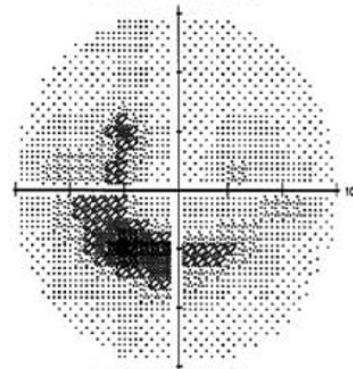

At 16 months

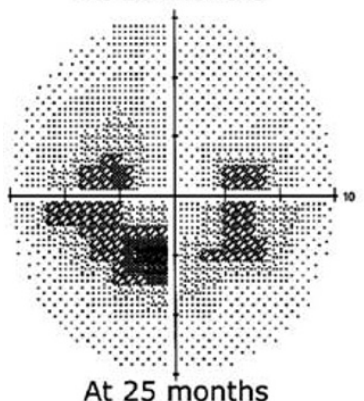

At 25 months
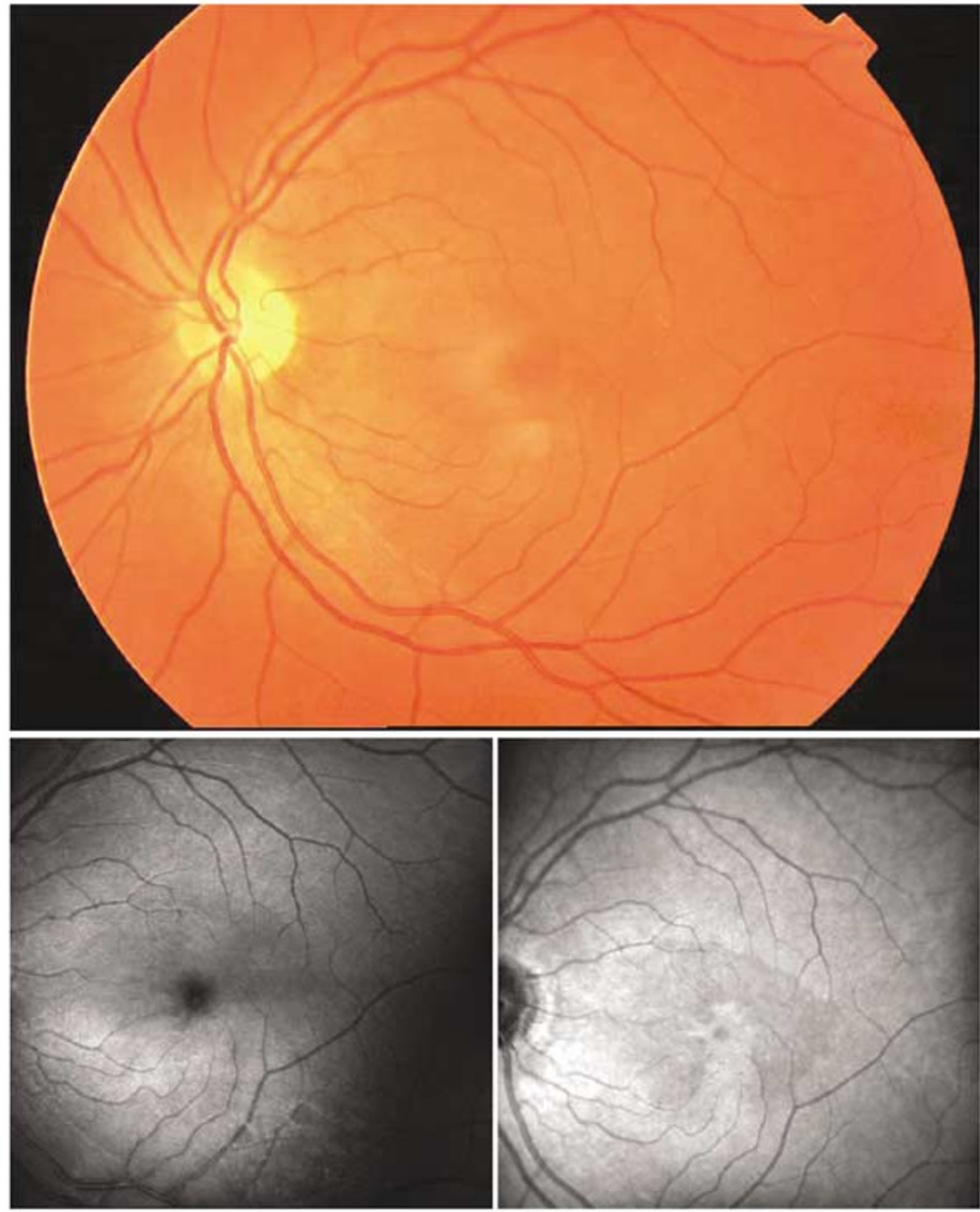

b

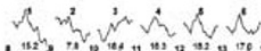

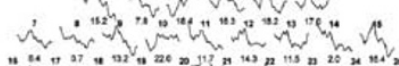
(N)

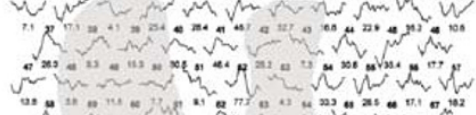

of

$\underbrace{}_{n=1}$

II

.

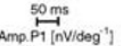
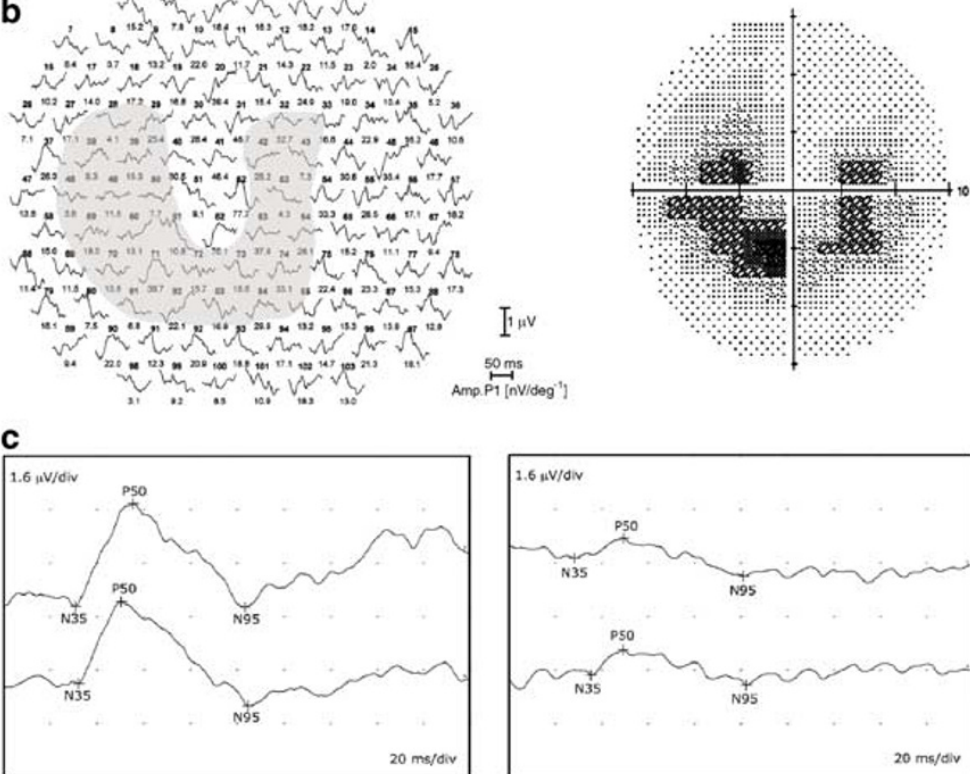

Figure 1 Left eye of patient 1. (a) Evolution over time of Humphrey automated VF (AVF) 10-2 TT; (b) all at 25 months after presentation: top, fundus photograph; second row red-free (RF) at left, and IR image at right; third row, mfERG (103 hexagons) at left, and Humphrey AVF 10-2 TT at right; and (c) pattern ERG of left eye at 16 months (left) and at 28 months (right). Note that changes are barely visible on white light and RF image, with superior visibility of U-shaped macular reflex on IR image; U-shape is congruous with the area of low-amplitude responses on mfERG and scotomata on AVF. 

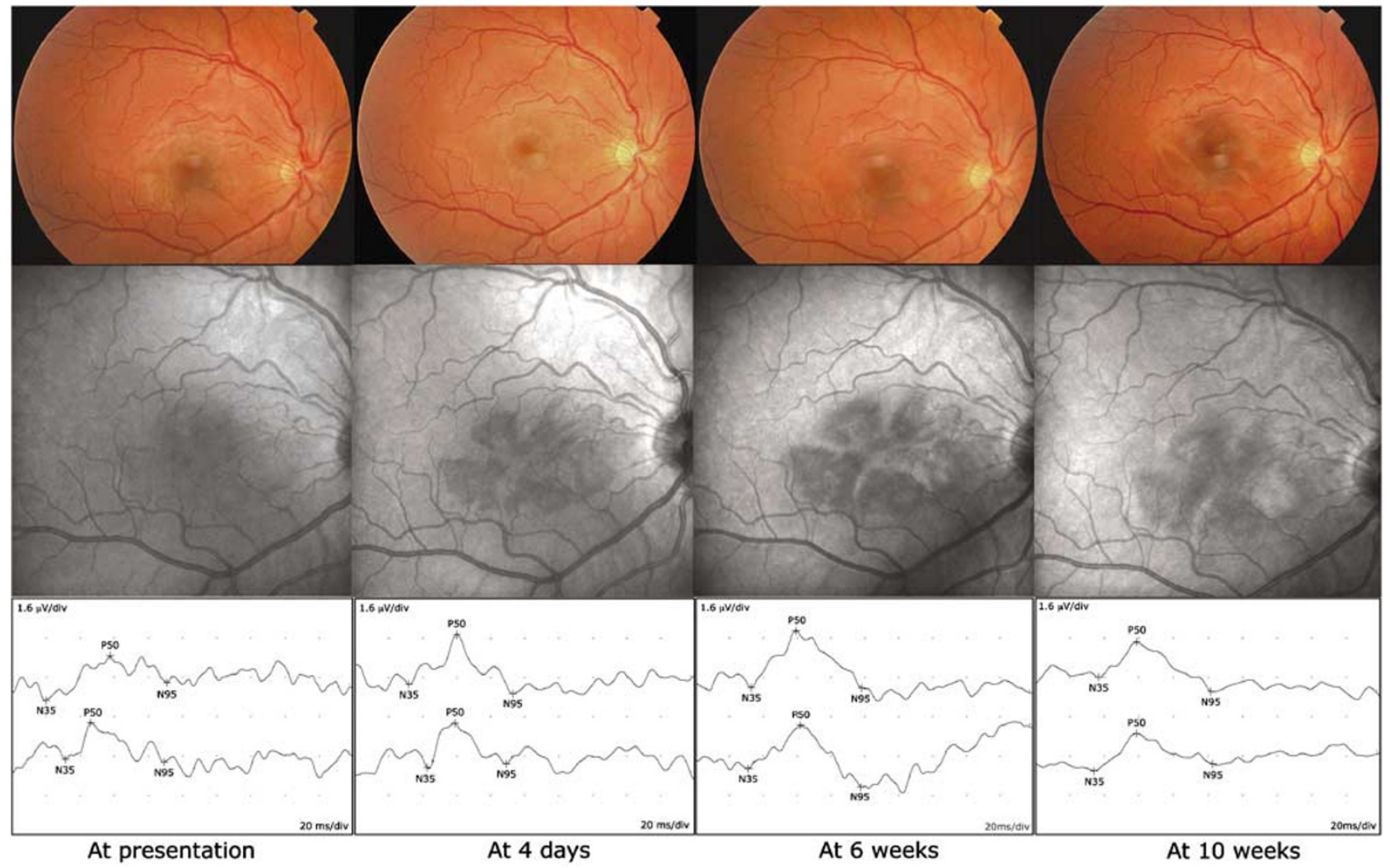

Figure 2 Right eye of patient 2 at different visits as indicated: top row, fundus pictures; middle row, IR images; bottom row, pattern electroretinograms. Note that whereas fundi and IR images are normal at presentation, macular function as depicted by PERG is severely disturbed, with reduction in both $\mathrm{P}_{50}$ and $\mathrm{N}_{95}$; later, function progressively normalises while IR images provide proof of maculopathy. 
pericentral area, followed by flickering lights and blurred vision in both eyes (BE). Subsequently, she was able to draw well-delineated, bilateral U-shaped scotomata on Amsler grid. A fluorescein angiography performed 4 days later was normal, although an abnormal foveal reflex was seen on red-free pictures. VF examination confirmed the presence of bilateral U-shaped paracentral scotomata. A normal brain MRI scan and EEG excluded central nervous system involvement. When seen 10 days later at our hospital, best-corrected visual acuity was 20/ 20 in BE. Slit-lamp examination and intraocular pressure were unremarkable. A discrete red-green colour vision defect was detected in BE. Fundoscopy revealed reddishbrown petaloid lesions in both macular regions, with some discrete small whitish drusen. The fundus lesions were best seen with monochromatic IR light (Figure 1). Goldmann perimetry and central 10-2 Humphrey VF confirmed the horseshoe scotomata in BE (Figure 1). The visual-evoked potentials to a pattern-reversal stimulus showed normal latencies and small amplitudes for all check sizes. Full-field flash electroretinography (ERG) and electro-oculography were normal.

At 16 and 28 months follow-up, mfERG showed a good central response, but paracentral regions had tiny amplitude recordings corresponding to the VF defects

(Figure 1). Vertical OCT3 scans suggested a thinned area in the corresponding macular region. After 16 months, pattern electroretinography (PERG) amplitudes and latencies returned to near normal, but amplitudes became much smaller again after 28 months (Figure 1).

Case 2 is of a 19-year-old woman with bilateral, central scotomata of acute onset, 4 days after a bout of influenza. When examined within $8 \mathrm{~h}$ after the onset of symptoms, only pericentral scotomata on perimetry and low amplitudes on PERG were noticed, fundoscopy and imaging were normal (Figure 2). After 3 days, function had improved to near normal, while IR imaging then showed bilateral horseshoe-shaped macular lesions (Figure 2). This situation remained unchanged over a period of 10 weeks.

\section{Discussion}

To our knowledge, there is only one previous report in the literature of two cases of AMNR occurring following postpartum hypotension. ${ }^{4}$ A follow-up of 28 months allowed collection of detailed longitudinal data on AMNR after C-section in the first case. Our patient suffered from constant hypotension with no documented acute drop in blood pressure prior to the onset of her complaints. However, she may have suffered from acute vasoconstriction in the chorioretina following the administration of methylergometrin, which is known to induce peripheral vasoconstriction and acute ischemia. ${ }^{9}$ Additionally, it increases vasoconstrictive effects of etilephrine, which was administered later on. It is therefore likely that AMNR in this patient was due to transient chorioretinal vasoconstriction. The IR pictures, mfERG changes, and OCT and PERG findings all support the view that the lesions in AMNR are most probably situated in the outer retina, as has been suggested by others. $^{10}$

The second patient's case illustrates the time lag between onset of visibility of macular lesions and recovery of function, in AMNR secondary to a viral illness. Indeed, it seems that fundoscopic lesions become apparent several days after the onset of the functional deficit and may remain visible after functional recovery.

Finally, the findings in both cases also suggest that IR imaging is superior to red-free or white light imaging.

\section{Acknowledgements}

We acknowledge the patients for their willingness to collaborate in this study.

\section{References}

1 Bos PJ, Deutman AF. Acute macular neuroretinopathy. Am J Ophthalmol 1975; 80: 573-584.

2 Gomez-Torreiro M, Gomez-Ulla F, Bolivar Montesa P, Rodriguez-Cid MJ. Scanning laser opthalmoscope findings in acute macular neuroretinopathy. Retina 2002; 22: 108-109.

3 Turbeville SD, Cowan LD, Gass JD. Acute macular neuroretinopathy: a review of the literature. Surv Ophthalmol 2003; 48: 1-11.

4 Stilma JS, de Lange JJ, Crezee FC. Bilateral central scotoma with preservation of central vision in 2 patients following caesarean section under spinal anesthesia. Doc Ophthalmol 1987; 67: 59-68.

5 Browning AC, Gupta R, Barber C, Lim CS, Amoaku WM The multifocal electroretinogram in acute macular neuroretinopathy. Arch Ophthalmol 2003; 121: 1506-1507.

6 Chan WM, Liu DT, Tong JP, Law RW, Lam DS. Longitudinal findings of acute macular neuroretinopathy with multifocal electroretinogram and optical coherence tomography. Clin Exp Ophthalmol 2005; 33: 439-442.

7 Maturi RK, Yu M, Sprunger DT. Multifocal electroretinographic evaluation of acute macular neuroretinopathy. Arch Ophthalmol 2003; 121: 1068-1069.

8 Shukla D, Arora A, Ambatkar S, Ramasamy K, Perumalsamy N. Optical coherence tomography findings in acute macular neuroretinopathy. Eye 2005; 19: 107-108.

9 Carrel T, Carrel B, Jutzi H, Nachbur B. Drug-induced acute arterial occlusion. Helv Chir Acta 1990; 57: 169-175.

10 Sieving PA, Fishman GA, Salzano T, Rabb MF. Acute macular neuroretinopathy: early receptor potential change suggests photoreceptor pathology. Br J Ophthalmol 1984; 68: 229-234. 